\title{
THE TEST SUB GROUP (TSG)
}

\section{A Cooperative Approach to Improve the Release Quality before Type Acceptance}

\author{
Giulio Maggiore $t$, Silvio Valeau $+t$, Josè Pons $++t$ \\ + CSELT, Centro Studi e Laboratori Telecomunicazioni \\ via Reiss Romoli 27410148 Turin ITALY \\ Giulio.Maggiore@cselt.it
}

\author{
++ TIM, Telecom Italia Mobile \\ largo Tassoni 323 Rome ITALY \\ svaleau@mail.tim.it
}

+++ Ericsson Eurolab Deutschland GmbH

Ericssonallee 152134 Herzogenrath GERMANY

Jose.Pons@eed.ericsson.se

\begin{abstract}
The development of telecommunication market has increased dramatically during the last ten years, forcing mobile operators to introduce new services for the customers. To do so, the time available for testing decreases drastically, forcing the operators to request always more quality to the manufacturers providing their own network elements. In this scenario, a group called Test Sub Group (TSG) has been created among the mobile operators using ERICSSON CME 20 Switching Systems, with the purpose to bring the operators' point of view inside Ericsson System Integration activities. In this phase, in fact, the possible improvements have lower costs for both, the supplier and the operators, and high effects on release software quality. In this paper, TSG and its way to work inside the Ericsson process is described, highlighting the benefits for Ericsson and the Operators. The paper continues addressing the methodology used and its application in the context of the Switching Systems R8 release with an example related to GPRS testing. A set of measurements to evaluate process and product quality is described. Some considerations about the validity of the "TSG" model are made, and its applicability to various market situations is analysed.
\end{abstract}

The original version of this chapter was revised: The copyright line was incorrect. This has been corrected. The Erratum to this chapter is available at DOI: 10.1007/978-0-387-35516-0_20 
Keywords: CME 20, End-to-End testing, TSG, GSM, GPRS

\section{INTRODUCTION}

In the last decade the development of the telecommunications had a tremendous growth. This development has resulted in new market opportunities and reduction in costs for the final users. The deregulation of the market has resulted in the entry of new players, as network operators and service providers.

The cellular communication arrived in the early 1980s marked a turning point in telecommunications. GSM and DCS 1800 are undoubtedly the major achievements in modern cellular solutions. Millions of customers use nowadays those voice services and look forward to adding non-voice services to their service portfolio. In fact with the new data services like WAP, GPRS, the idea of "Wireless office" is a reality. Wireless means you can get the information any time and any place.

In this scenario, GSM network operators need to put in service high quality network elements, sooner than in the past.

In this paper, the Ericsson CME 20 TSG Switching System ${ }^{1}$ (SS) mobile operators' experience is described. Inside CME 20 User Group, a subgroup called Test Sub Group (TSG) was formed in January 1999 to support Ericsson in increasing the quality of its SS equipment's, and consequently to have an earlier roll out for the operators. This is a work in progress paper so no results have been reported yet. The paper is structured as follows. The section 2 explains what is TSG, how it works in the Ericsson industrialisation process, its reasons and goals, why it represents a win-win situation between Ericsson and the Operators. The section 3 gives the TSG SS methodology. The section 4 reports the current project organisation, named "Tornado", referred to R8 release of the Switching System's nodes and the measurements proposed to evaluate $\mathrm{R} 8$ process and product quality. In the section 5 the validity of the TSG model is addressed.

\footnotetext{
${ }^{1}$ In the TSG Switching System are included MSC/VLR, HLR, SSF, SCP and in this specific case the GPRS packet nodes.
} 


\section{THE TEST SUB GROUP (TSG) SWITCHING SYSTEMS (SS)}

Starting from the positive experience of the TSG BSS, made inside CME 20 for the BSS R7, in January 1999 a group of operators, Mannesmann Mobilfunk, Swisscom Mobile, Telenor Mobil, Telia Mobile, TIM, Vodafone UK, Libertel, and later on in the project Pacbell Wireless, proposed to Ericsson to launch, under the hat of CME 20 User Group, a TSG for the next SS release. Ericsson saw this new activity as an opportunity to improve its own systems. In this section what is the TSG and how it works in the Ericsson process is reported, what the TSG reasons and goals are, and why it is considered as "win-win" situation between Ericsson and the Operators.

\subsection{What is TSG}

The TSG is a group operating on behalf of the CME 20 User Group, during the Ericsson system integration phase of a release, in order to have a faster acceptance and rollout and, consequently to obtain a high quality release delivered at the end of the industrialisation process. The purpose of the group is to provide feedback, from the operator's point of view, to the Ericsson system integration phase. The TSG does not provide any requirements that Ericsson has to fulfil, and any constraints, which can cause some delay in the delivery date. It will be an Ericsson choice to implement or not the comments received according to its own policy. Quality is meant as the service perception seen from the operators' point of view, which means the ability to use a service, to manage the network elements and to be able to collect all the statistics, measurements and billing information.

\subsection{The TSG SS in the Ericsson Process}

The Ericsson's test process, consists of two main activities:

- Global Application System (GAS) testing activity, under the responsibility of a central Product Unit, in which is made the testing of all the world common parts of the new release;

a Market Application System (MAS), under the responsibility of the Ericsson's local company, in which the testing of all the market adaptations is made.

Both Ericsson and the CME UG (User Group) have identified a need for Generic Switching System Testing which is performed on the GAS part.

TSG Generic testing has the objective to check the common part of the release (GAS). By doing so, the operators share resources in analysing the 
GAS testing, and Ericsson has an early feedback, which in the past was supposed to be received only during the type acceptance ${ }^{2}$ activities.

A higher level of quality is achieved earlier in the life span of the release and the individual Ericsson local offices execute less repetitive testing for the respective customers.

The scope of a generic testing phase is limited to core CME 20 and common functionality across the CME UG operators. It remains the responsibility of the local Ericsson companies to prove national or customer specific software package and features.

The testing is carried out on a generic network environment. This environment includes as many customer specific settings as possible. TSG aims, together with Ericsson, to reach an agreement on identifying a suitable environment. The test environment is as close as possible to an end-to-end environment so that the whole product can be verified.

The parameter settings for the generic network environment are as close to the customer recommended setting as possible with as many functions as possible switched on. This recommendation is done jointly by Ericsson and TSG. The TSG has access to the parameter settings to enable individual members to assess the impact of their own parameter requirements on the generic test results.

TSG does not want to change Ericsson's way of working: Ericsson is not expected to produce extra documentation because of TSG more than what is produced anyhow. TSG just simply analyses, and gives feedback to the Ericsson work procedures. It is Ericsson's choice to make changes into the methodology using the TSG suggestions. It is in the interest of Ericsson to incorporate the TSG suggestions to increase the quality of the Ericsson products.

\subsection{The New "Supplier-Customer" Process}

The "Supplier-Customer" process that TSG is supposed to modify has two separated phases:

- The Supplier's phase, in which the new product is designed and integrated;

- The Customer's phase in which the product is tested against the internal requirements and then if accepted rolled out in the network.

In this way, any problem or a not completely satisfactory feature in the new product, is found when the Supplier's design and integration process is

${ }^{2}$ The type acceptance activity is made by all the testing aspects of a new product/release prototype, whose acceptance implies the acceptance for all the instances of the same product/release. 
finished, and the same product has been delivered to various customers. The possible modifications on the product has to be done on several packages or postponed to a next release. From the customer's point of view this means as the best option, to have a delay in the roll out phase or even worse to postpone the delivering of the new service to a next product's release.

The new "Supplier-Customer" process due to the TSG interaction, see the Customers involved since the Supplier's System Integration phase, giving early feedback to the Supplier, that can possibly modify the product ones and for all the customers. This early involvement allows to the operators to get more product's information that can help reducing type acceptance and roll out and consequently the whole time to market.

\subsection{The Win-Win Situation}

The TSG activity is a joint venture between Ericsson and the CME User Group. The basis for the joint venture is built upon the expectation of reaching a mutual benefit, which results in a win-win situation for both parties. For example it is expected that members of the Test Sub Group perform the First Office Application (FOA). In other words, FOA operators are expected to be interested to participate actively in TSG. This is only one example of possible win-win situation, in which operators have an early roll out and Ericsson a better knowledge of the FOA site.

Both parties define in detail in the Joint Project Specification for each release what are the minimum benefits for the process to be undertaken. These are specified as measurable goals jointly valid for Ericsson and TSG members.

\subsubsection{The TSG benefits as seen by the Operators}

A successful TSG may result in a reduction of the Type Acceptance and roll out time for each operator, with an increased release quality. This results in a measurable cost saving for the operator.

At General Availability, the date at which the products are available for all customers, the operators receive products from Ericsson that have been tested according to the operator's point of view, prior to releasing the product for customer acceptance.

By doing this, the operators are in the position to accept Ericsson products sooner and doing this reduces trouble shooting on the new release. The customers also have the possibility to join efforts and resources among themselves, since they have been co-operating from early stages of the project already. 
The TSG operators actively strive to find synergies among themselves by identifying common Test Objects that will result in a reduction of the time used to verify the different Market Application Systems.

By providing early information to the operators the rollout can be prepared early.

\subsubsection{The TSG benefits as seen by Ericsson}

A successful TSG may result in a reduction of Ericsson Time to Customer, allowing to use the results of the report that is written by TSG at the end of the project, towards customers outside the CME User Group.

Ericsson has an early involvement of the Supply organisations for those operators that are members of TSG during the verification phase, by creating an early awareness of the products within TSG operators and the corresponding Ericsson Local Offices.

A successful TSG activity will reduce the time and activities for Type Acceptance and roll out for the total Ericsson markets. This will be checked as resulting from the normal Type Acceptance activities and specially for TSG customers. This is measured on a project basis together with TSG customers. Ericsson delivers products with better quality since they have been tested early following the operator's point of view.

Ericsson staff (testers etc.) involved in the TSG activities meet the customer on a technical level. By that, they get an understanding of how the product quality impacts the customer and by that how important their own performance is. Additionally they have access to the verification competence available with the operator's technicians [1].

Realistic "customer-like" configurations are used when testing. This has a positive effect on the rollout as more "configuration-specific" faults are found before the Roll Out.

The test and verification staff gains a more customer oriented attitude which results in a more customer oriented way of working at an early stage of the production process then as it was the case before. The TSG effort and results are documented in agreement with Ericsson.

No results are available on both Ericsson's and Operators' side, because this is a work in progress paper. 


\section{THE TSG SS METHODOLOGY}

The TSG SS methodology is based on the End to End methodology defined in the EURESCOM P412 [2] and $\mathrm{P}^{2} 13^{3}$ project [3] applied to the operator's operational environment. The application of the End-to-End methodology allows to check the service, since the very early stages of the products availability, checking not only network elements (NEs) functionality in the new release, but also the interactions with the NEs in the previous release.

All the aspects of the service functionality are checked together with network operability aspects, e.g. O\&M, billing and statistics and measurements verifications. All these verifications, performed together, allow to check properly the services functionality as well as operators do in their own network. All this verification will increase the release quality, allowing speeding up the type acceptance and the roll out activities.

\subsection{The End-to-End Methodology}

In general, the object of the End to End methodology is the set of two Network Elements connected directly (or possibly through a third transit network element, or a sequence of transit NEs), as described in figure 1 . The abstract testing methods used is Multi Party Testing Method as described in [4].

The networks are observed and controlled through some interfaces (usernetwork or network-network) which are called PCOs (Point of Control and Observation) [5]. There are at least two PCOs for the control and observation at the external borders (minimum mandatory configuration) and, as an optional feature, one or more additional PCOs only for observation (i.e. in order to monitor the internal network behaviour).

The actual number and location of the PCOs depends on the characteristics of the networks and on the functionality to be tested. The physical testers shown in figure 1 are logically located at such Points of Control and Observation. Such "points", in real life testing, may actually be close to each other or may possibly be very far: actually End to End may be applied to geographically distributed testing.

The monitoring point, indicated in the figure 2 , is optional. The two other PCOs must always exist; as a particular case, the functions of one tester

${ }^{3}$ EURESCOM P613 project defined methodology and End to End test specifications written in Concurrent TTCN [TTCNV2_99] for the GSM-ISDN-PSTN Network Integration Testing. Projects results are publicly available on http://www.eurescom.de/public/Projects/P600-series/P613/P613.HTM 
located in one of such PCOs might actually be replaced by equivalent actions performed by the corresponding node.

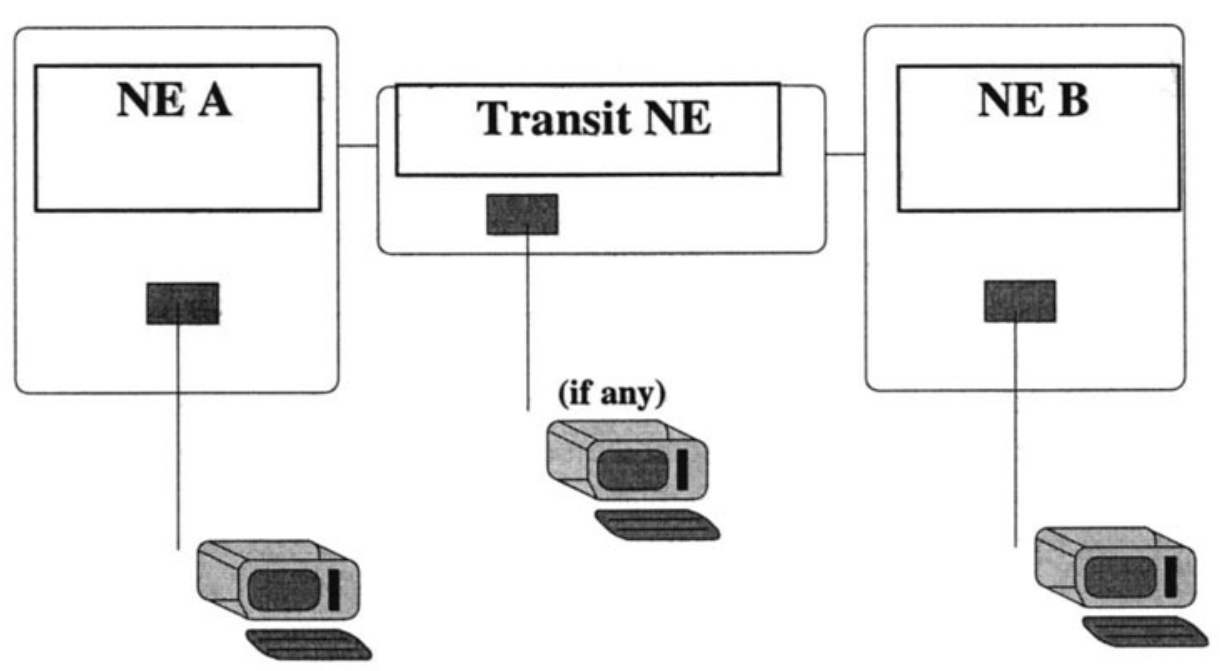

Figure 1. Generic configuration for an End to End testing session, including distributed Testers

In End to End, the network is tested as it is seen from the user's Terminal Equipment (TE), i.e. taking the user-network interfaces as PCOs (Point of Control and Observation). For example, A = GSM Access protocol, $\mathrm{B}=$ an ISDN Basic or Primary Access protocol.

The "End to End Network Under Test" is composed of all the parts that contribute to perform the expected network functionality, i.e. connection and transport of data and signals between the external gates (A, B, [M]):

- protocols that manage the external entities connected to the System Under Test (SUT) (access protocols in the case of End-to-End testing);

a each network component or function involved in a call between the Aside and the B-side (e.g. all call-control functions in all crossed NEs).

$\mathrm{EtE}$ is concerned only with the external behaviour of the network. For a complete description of the methodology refer to [1]. 
Network Under Test

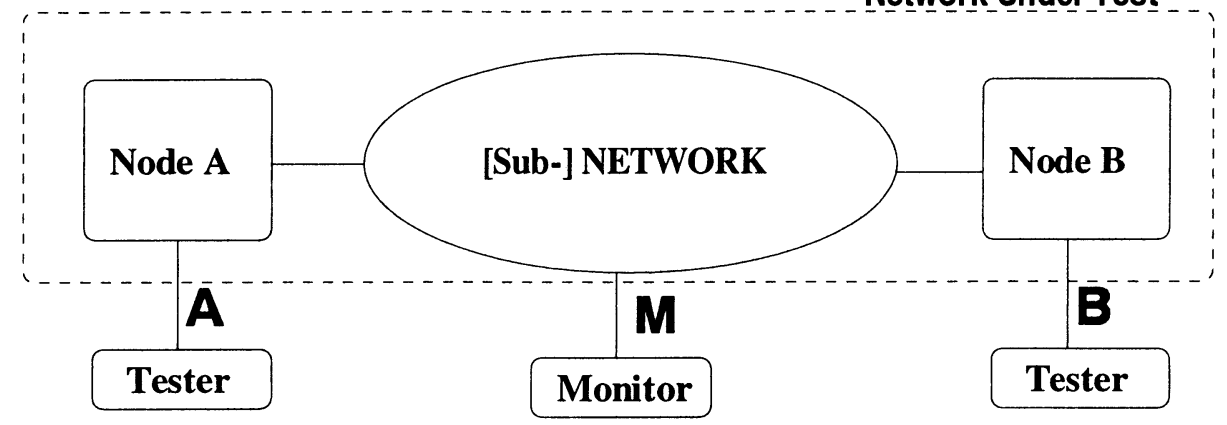

Figure 2. Example of a System Under Test (SUT), from the End to End point of view

\subsection{Examples of Application of End-to-End Methodology}

Examples of the aspects listed above are the worlds of ISDN, IN, GSM. For example in the case of a data call between a GSM user belonging to a PVN and an ISDN user, several network features are involved:

a switching functions (LE, TE, MSC);

口network databases (HLR);

anodes containing service logic (SCP).

When each network element is tested separately, there is no confidence on the correct behaviour of the whole network. This is particularly valid for those services, whose implementation requires the interaction between NE based on telecommunication (TLC) technology with NE based on information technology (IT). This is exactly the case of the new GPRS service, whose deployment requires TLC NEs (BSS, MSC, HLR, and so on) and IT NEs (SGSN and GGSN). In fact as shown in figure 3, where is represented the ETSI GPRS functional architecture, there are quite a lot of interactions between TLC and IT NEs. Using the End-to-End methodology, in order to have a clear picture of the overall GPRS functionality, Terminal Equipment (TE) will be simulated on radio interface (Um) and on data network (Gi), and monitor points will be placed on the all the $\mathrm{G}$ interfaces. 


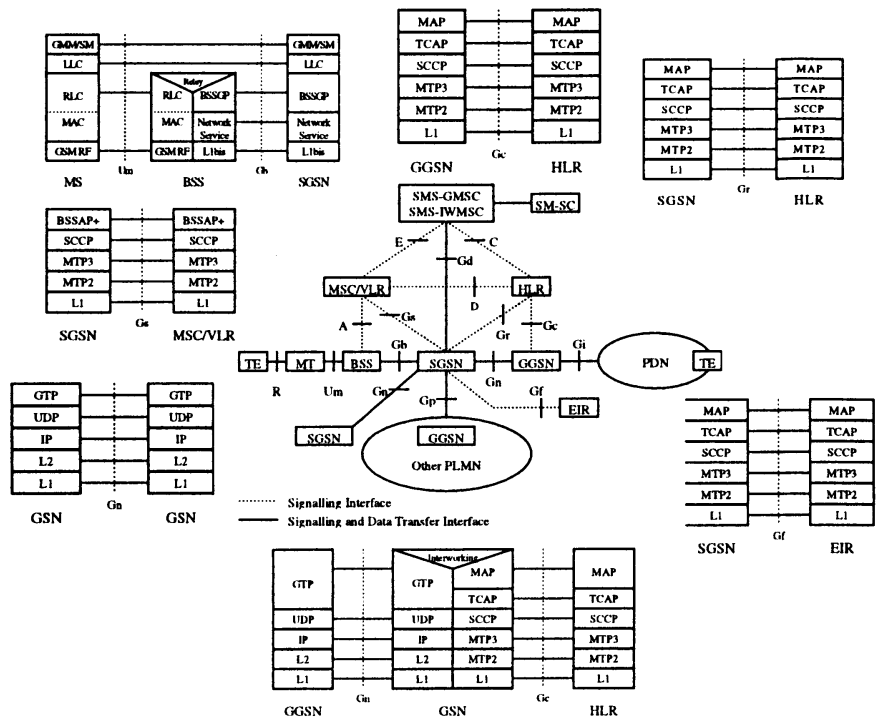

Figure 3. Overview of the GPRS Logical Architecture and protocol stacks

\subsection{TSG SS R8 End to End Application}

The application of the End to End methodology considered inside the TSG SS R8 can be summarised with the following items:

口 Simulate access features, using protocol analyser, real terminal or network elements which provide the access facilities;

- Monitor network features, using monitor facilities, by applying End to End stimulus to use the service, as seen in the previous point.

a Check within each test case executed not only the telecommunication service from the protocols point of view, but also all the other aspects relevant for the operators like:

- Charging;

- Statistic measurements (STS, routes, traffic dispersion measurement);

- Operation and Maintenance.

- Only when all these aspects are correct, an End-to-End test case can be declared PASS. 


\section{THE TORNADO PROJECT}

Inside the R8 project, Ericsson decided to set-up a project addressing the TSG activities. This project was called "Tornado". The name was chosen to highlight the Ericsson's awareness of the customer's presence inside the project. In this section the project organisation will be described.

\subsection{The Project Organisation and Phases}

The project has been organised under Ericsson's responsibility, and follows Ericsson's system integration phases: features analysis, Test Description design, test case execution. These phases have been mapped into set-up phase, analysis phase and test execution phase inside the Tornado project.

\subsubsection{Set-up phase}

After the development of the project terms of reference together with the operators, Ericsson presented the new features to be developed and verified in the new release and the customers made a choice on the list of interesting features. A selection of feature was necessary because of a limitation of resources. For the remaining features not selected by TSG, Ericsson tried to apply the TSG methodology. A detailed time plan with deadline for the feedback was made, in order not to delay the ongoing project with late customer comments or suggestions. Agreements have been made with the Ericsson Local Companies to get them involved in the project as soon as possible. The Ericsson development and verification Switching System Project was informed about the direct customer involvement and its implications, since this was done for the first time for the Circuit Switching Systems Product Unit.

\subsubsection{Analysis phase}

In the analysis phase TSG operators provide feedback to the Test Descriptions (TDs) written by Ericsson for the release features verification. The specifications used as a reference for commenting the TDs are the ETSI standards and in case of proprietary services Ericsson Functional Specifications (FSs).

A common understanding is to have preliminary information provided by Ericsson. In this phase early information on TDs allow to TSG operators to 
give feedback that can be endorsed by Ericsson with low impact on the process.

The suggestions made by the operators are considered by Ericsson case by case and incorporated into the documentation.

The experience done, highlight minimal delays due to the customer involvement and valuable customer comments. The usage of Internet as the media to exchange documentation and information in Tornado Project optimise the project activity and reduce the project disturbance.

Face to face meetings are arranged when needed.

Ericsson provides to TSG operators, also documents relevant for the roll out, such as parameter information, information on changes in the Operation and Maintenance documentation. To all the documents TSG operators give feedback, highlighting the aspects to be improved and the relevant aspects from the operators' point of view.

\subsubsection{The TSG SS feedback}

As previously described, TSG is a "Win-Win" situation between Ericsson and the operators. This means that TSG doesn't put constraints to the Ericsson plan, in order not to delay the product delivery and consequently operator's time to market. The TSG SS analyses test architectures and specifications, and all the documents useful for the roll out of the new release, and gives feedback to Ericsson. It will be Ericsson's choice to endorse or not the comments received, on the basis of its own development policy, strategy and so on.

\subsubsection{Test execution inspection}

The test case execution phase starts after the analysis phase and the production of the final TDs on the Ericsson side. This phase intends to give to the TSG operators an early feeling of the services in the new release and the evidence of the System Integration process executed according with the reviewed documents. The position of TSG is being an observer. The feeling perceived in this phase together with the measurements taken during the project will help TSG operators in writing the final report, expressing opinion on the process and product quality.

\subsubsection{Final report}

All the activities performed by TSG operators together with Ericsson will be written in a final report. This TSG document represents the experience of the TSG operators related to the release quality and the Ericsson process 
applied for it. The final TSG opinion is built up on the bases of the measurements collected during the project and the service perception of the TSG operators during Ericsson test cases execution. The impact of TSG feedback on the Ericsson work is also evaluated.

This report is supposed to be delivered by TSG in preliminary version two weeks after the Ready for Service Date. A final version will be available two weeks after General availability Date. At the end of the roll out activity, the TSG operators will once more review the report. All these versions will be available inside the CME 20 user group.

\subsection{The Measurements Proposed}

In order to collect some figures to be used together with the feeling perceived during test cases executions, TSG and Ericsson agreed on a set of measurements. In some cases comparison between R8 and R7 will be attempted, in order to highlight positive or negative trends between the two releases. The proposed measurements are mainly taken from EIRUS ${ }^{4}$ [6], but also other measurements are taken.

\subsubsection{What TSG SS is going to measure}

During R8 project, TSG SS is going to measure:

口Ericsson Process established for R8;

口Ericsson R8 Products.

Measuring Ericsson process TSG would like to keep track of the information flow, which is going to set-up the R8 products. Measuring R8 products TSG is going to measure the quality perceived, in terms of "trouble

4 EIRUS is E-IPQM and E-RQMS User Group (http://www.eurescom.de/ publicwebspace/P300-series/P307/eirus/rules.htm). The objectives of the group are:

a The implementation of E-IPQM and E-RQMS;

a Discussion in order to find best solutions and practices;

L Learn from the experiences of other companies;

a Formulate a consensus on change requests to E-IPQM and E-RQMS in order to preserve uniformity;

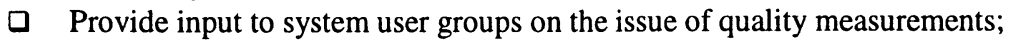

a Maintain contacts between PNOs and Suppliers;

- Establish a common attitude towards quality;

a Promote the knowledge about E-IPQM and E-RQMS.

- Communicate with various institutions and organisations in order to exchange ideas.

Decide on actions which improve the internal organisations of EIRUS and its activities in general. 
report", during the Ericsson test executions and afterwards during type acceptance and roll out.

\subsubsection{How TSG wants to measure process and products}

For the Process measurements will be considered the following indicators:

口Milestone delay (EIRUS);

口Requirements and Design Stability (EIRUS);

口Test Tracking (EIRUS);

-Action Points Delay (to TSG);

Documentation Delay (to TSG);

For the Product measurement will be considered:

$\square$ Trouble report per service;

口Trouble Reports comparison per test phase;

$\square$ Trouble Reports comparison per life cycle.

In the following subsections each measurement will be described.

\subsubsection{Milestone Delay}

Milestone status metrics can help the suppliers in assessing the robustness of their software process by monitoring key milestone schedules. Frequent occurrences of milestone slip may indicate planning, resource allocation, testing and other software quality related problems. These metrics can help the suppliers to better understand and control the Ericsson's milestone delays that are visible to the customers through customer interactions.

Data in input to TSG:

口List of INDUS R8 project milestones;

$\square$ Planned completion for each milestone;

$\checkmark$ Actual completion date for each milestone.

The Milestone Delay will be calculated as:

Milestone Delay $=($ Actual date - Planned Date $)$ for each milestone

\subsubsection{Requirements and Design Stability}

Excessive number of changes, additions and deletion to requirements, design, code and test cases can adversely impact release schedules and software quality. Minimising and controlling these changes, the supplier can improve software quality and customer satisfaction. The TSG application for R8 will be related only to addition and deletion of features.

Data in input to TSG: 
Original number of Features originally planned;

$\square$ Total number of deleted features;

$\square$ Total number of added features.

So the Requirements and Design Stability can be calculated as

$$
\text { StabilityIndex }=\frac{\text { FeatureChanges }(\text { addition, deletion })}{\text { FeatureChanges }+ \text { FeaturesInitialPlanned }} \%
$$

\subsubsection{Test Tracking}

Software testing plays a very important role in improving software reliability by removing defects and verifying performance before the release of a software package to the customers. These metrics will provide the suppliers with data by which they can assess the progress of their test planning and test activities. The information is used to track testing activities during integration, regression and system testing life cycle phases.

Data in input to TSG:

- Total number of Test Cases Planned (P);

- Total number of Test Cases Executed (E);

- Total number of Test Cases Passed (PA).

The test tracking metrics are the following:

- Percentage of Planned Test Execution (PTE):

$$
P T E=\frac{E}{P} \%
$$

- Percentage of Yield of Executed Test (YET):

$$
Y E T=\frac{P A}{E} \%
$$

- Pass percentage based on Tests Planned (PTP):

$$
P T P=\frac{P A}{P} \%
$$


The thresholds fixed by EIRUS for these parameters are:

a PTE

$$
P T E \geq 97 \% \Leftrightarrow \text { GreenCondition }
$$

90\% $\leq$ PTE $\leq 97 \% \Leftrightarrow$ YellowCondition

$P T E \leq 90 \% \Leftrightarrow \operatorname{Re} d$ Condition

口 YET

$$
\begin{aligned}
& Y E T \geq 95 \% \Leftrightarrow \text { GreenCondition } \\
& 85 \% \leq Y E T \leq 95 \% \Leftrightarrow \text { YellowCondition } \\
& Y E T \leq 85 \% \Leftrightarrow \operatorname{Re} d \text { Condition }
\end{aligned}
$$

口 PTP

$P T P \geq 92 \% \Leftrightarrow$ GreenCondition

$77 \% \leq P T P \leq 92 \% \Leftrightarrow$ YellowCondition

$P T P \leq 77 \% \Leftrightarrow \operatorname{Re} d$ Condition

\subsubsection{Action Points Delay}

Frequent occurrences of action points slip might be the indication of problems in the release progress, or in the supplier process.

Data in input to TSG:

- Action points planned dates;

- Action points Actual dates.

$$
A P D=\text { Actual }- \text { Planned }
$$

\subsubsection{Documentation Delay (to TSG)}

As indicated also for the Action Points Delay, frequent occurrences of documentation delay might be the indication of problems in the release progress, or in the supplier process. The documentation considered is described in the project specification and the Documentation Delay will be measured as:

$$
\text { DD = Actual }- \text { Planned }
$$




\subsubsection{Trouble report per service}

Ericsson usually writes trouble reports (TRs) per software block. To be able to make a comparison between two releases (e.g. R7 and R8), TSG proposed to group TRs in order to create containers that can be compared. Each container can be a service or a group of services belonging to the same area (e.g. CCS\#7, ISDN, etc.). For each service or group of services will be established a priority, which can be High, Medium, Low. The regression test will be considered as a big group, because of the importance it has for the operators. The services mentioned are evolution or improvements from the previous release. Examples are:

口 Regression Test (High);

口 Service 1 (High priority);

口 Service 2 (Medium priority);

口 Service 3 (Low priority).

This grouping can allow the comparison as showed in the table 1

Table 1. TR per serrvice comparison between R7 and R8

\begin{tabular}{|l|l|l|}
\hline & R7 & R8 \\
\hline $\begin{array}{l}\text { Regression } \\
\text { (High) }\end{array}$ & $\begin{array}{l}\text { TR } \\
\text { (A,B,C) }\end{array}$ & $\begin{array}{l}\text { TR } \\
\text { (A,B,C) }\end{array}$ \\
\hline $\begin{array}{l}\text { Service 1 } \\
\text { (High) }\end{array}$ & Phase 1 & Phase 2 \\
\hline $\begin{array}{l}\text { Service 2 } \\
\text { (Medium) }\end{array}$ & TR(A,B,C) & TR(A,B,C) \\
\hline
\end{tabular}

In order to have a fair comparison between the two releases, for each service or group of services will be considered the number of the included blocks, in order to make an average that will be used for the comparison between the two releases.

\subsubsection{Trouble Reports comparison per test phase}

With this measurement, for each test phase inside the system integration process, will be made a comparison between the release under test and the previous one. Trouble Reports are normalised using the test cases executed. The Delta value will give the indication about the increasing or reducing of the percentage of Trouble Reports per test cases. The figures in the table 2 show that for phase 1 and 2 the new release has better values than the previous one. This is not the case of the phase 3 that has to be analysed.

Table 2. TR/TC per phase comparison between Release $\mathrm{N}$ and Release $\mathrm{N}+1$

\begin{tabular}{|l|c|c|c|}
\hline \multicolumn{1}{|c|}{ Project } & $\begin{array}{c}\text { TR/TC (\%) } \\
\text { Phase 1 }\end{array}$ & $\begin{array}{c}\text { TR/TC (\%) } \\
\text { Phase 2 }\end{array}$ & $\begin{array}{c}\text { TR/TC (\%) } \\
\text { Phase 3 }\end{array}$ \\
\hline Release N & 2 & 10 & 1.5 \\
\hline Release N+1 & 1.5 & 8 & 2 \\
\hline Delta & -0.5 & -2 & 0.5 \\
\hline
\end{tabular}




\subsubsection{Trouble Reports comparison per life cycle}

With this measurement, a projection outside the system integration phase is attempted. In fact Trouble Report found during system integration, are compared with those found during the First Office Application (FOA), and after 6 months rollout.

A comparison is also made between the last and the previous release (table 3).

Table 3. TR per life cycle comparison between R7 and R8

\begin{tabular}{|l|c|c|c|}
\hline \multicolumn{1}{|c|}{ Project } & $\begin{array}{c}\text { TRs System } \\
\text { Integration }\end{array}$ & TRs FOA & $\begin{array}{c}\text { TRs } \\
\text { GA }+6 \text { months }\end{array}$ \\
\hline R7 & TR $(\mathrm{A}, \mathrm{B}, \mathrm{C})$ & TR $(\mathrm{A}, \mathrm{B}, \mathrm{C})$ & TR $(\mathrm{A}, \mathrm{B}, \mathrm{C})$ \\
\hline R8.0 & TR $(\mathrm{A}, \mathrm{B}, \mathrm{C})$ & TR $(\mathrm{A}, \mathrm{B}, \mathrm{C})$ & TR $(\mathrm{A}, \mathrm{B}, \mathrm{C})$ \\
\hline Delta & TR $(\mathrm{A}, \mathrm{B}, \mathrm{C})$ & TR $(\mathrm{A}, \mathrm{B}, \mathrm{C})$ & TR $(\mathrm{A}, \mathrm{B}, \mathrm{C})$ \\
\hline
\end{tabular}

\subsection{The Present Status of the Project}

The TSG SS R8 project launched in January 1999 has reached at the moment half of its planned activities. The methodology chosen has been applied to provide feedback on test descriptions, and half of the comments provided to Ericsson have been implemented.

The future actions will be the test cases execution monitoring phase, collection of TRs and the reporting of the final consideration in the TSG Final Report. A clear picture of the overall benefits of the TSG approach will be seen only after the rollout of the R8 release, when product quality will be evident and the ratio quality/costs will be drawn for both the supplier and the operators.

\section{VALIDITY OF THE TSG MODEL}

The TSG SS model applied inside Ericsson CME 20 User Group, can also be valid for those operators, whose network or most of it, is provided by one supplier. In this case this model is fully applicable and the operators can get more confidence on the new release, with the exception of the verification of the market customisations. In fact the custom solution cannot be proved for obvious reasons in a co-operative environment.

The TSG model is also applicable when such a NE typology (e.g. all the MSC/VLR, HLR, SCP etc.) belong to the same supplier. In this case the operators can be confident of the new release with the exception of the compatibility tests between the NE from one supplier and NE from another 
supplier. So in this case a special compatibility testing session will be required during the type acceptance phase.

There is a third case in which the model is hardly applicable. It is the case when different suppliers provide the same NE type. In this case it could be very difficult to establish a co-operative approach for confidentiality reasons. Even if the model is applied with one supplier, a full Network Integration Testing Campaign should be issued to make sure everything works together. In any case this point needs to be experimented.

\section{CONCLUSION}

Customers are fundamentally changing the dynamics of the market place. The TSG is one example of a market become a forum in which customers play an active role in creating and competing for value. The distinguishing feature of this new marketplace is that customers become a new source of competence. The competence that customers bring is a function of the knowledge and skill they possess, their willingness to learn and experiment, and their ability to engage in an active dialogue. This will carry the market to develop a product shaped to the user needs, and not to the other way round. The products has to evolve in a way that enables future modifications and extension based both on customers' changing needs and companies' changing capabilities. Whether or not TSG has accomplished to his task will be discovered at the end of the R8 roll out, when pros and cons will be highlight for Ericsson and the Operators.

\section{References}

[1] C.K. Prahalad, V. Ramaswamy "Co-opting Customer Competence" Harvard Business Review January-February 2000.

[2] EURESCOM P.412 Deliverable 3: "Guidelines for NIT session management: volume 2: Guidelines and proformas".

[3] EURESCOM P613 Deliverable 3 "Test Specification for narrowband services (GSM, ISDN, PSTN)"

[4] ETSI ETR 193 "Methods for testing and Specification (MTS); Network Integration testing (NIT); Methodology aspects; Test Co-ordination Procedure (TCP) style guide".

[5] ISO/IEC 9646 (1994): "Information technology - Open Systems Interconnection Conformance testing methodology and framework

[6] E-IPQM and E-RQMS User Group (http://www.eurescom.de/ public-webspace/P300series/P307/eirus/rules.htm). 


\section{ABBREVIATIONS}

CME 20

DCS

EtE

ETSI

FOA

FS

GAS

GGSN

GPRS

GSM

HLR

IN

ISDN

ISUP

IT

LE

MAS

MSC

NE

$\mathrm{NtN}$

O\&M

PCO

PVN

SCF

SCP

SGSN

SS

SSF

STS

SUT

TD

TE

TLC

TR

TSG

VLR

WAP
Ericsson GSM Network product

Digital Communication System

End to End

European Telecommunication Standard Institute

First Office Application

Functional Specification

Global Application System

Gatway GPRS Support Node

General Packet Radio Services

Global System for Mobile communication

Home Location Register

Intelligent Network

Integrated Services Digital Network

ISDN User Part

Information technology

Local Exchange

Market Application System

Mobile Switching Centre

Network Element

Node to Node

Operation and Maintenance

Point of Control and Observation

Private Virtual Network

Service Control Function

Service Control Point

Serving Gprs Service Node

Swithing System

Service Switching Function

Statisctic and Traffic measurement Subsystem

System Under Test

Test Description

Terminal Equipment

Telecommunication

Trouble Report

Test Sub Group

Visitor Location Register

Wireless Application Protocol 